\title{
Linguistic Analysis Methods for Studying Small Groups
}

Iris Howley, Elijah Mayfield, \& Carolyn Penstein Rosé

Carnegie Mellon University

\section{Introduction}

Linguistic analysis of collaboration is a research area with stakeholders from multiple fields, including learning sciences, organizational behavior, sociolinguistics and other areas of linguistics, language technologies and machine learning, social psychology, and sociology. Each field brings with it its own valuable store of wisdom as well as its own sets of research questions and methodologies that bring out different subsets of facets. While we view collaboration from a multi-disciplinary perspective, we adopt a technical definition of what linguistic analysis is, specifically an analysis that employs constructs from the field of linguistics that are designed to study language as a primary focus of inquiry. While a wide variety of valuable frameworks for studying the language of collaboration have been developed within the learning sciences community, instead of considering these linguistic analyses of collaboration per se, we consider them applications of theoretical constructs from the learning sciences to language interactions. The focus is on learning processes viewed through language rather than the language processes themselves. The advantage of a linguistic framework is that it allows us to avoid making a commitment to one specific theoretical perspective within the learning sciences.

Within the broad field of linguistics, we focus on a specific subcommunity referred to as Systemic Functional Linguistics. The field of systemic functional linguistics is a largely descriptive linguistic tradition that provides a firm foundation in analyses of genres of writing or text-based interaction (Martin \& Rose, 2007; Martin \& White, 2005), as well as face-to-face interaction (Veel, 1999), characterized in terms of the choices authors and speakers make about how to present themselves through language (Halliday, 1994). The choices that authors and 
speakers make are mapped out in decision tree representations referred to as systems, which is where it gets its name. The constructs coming from the field of systemic functional linguistics provide us with a common foundation for exploring stylistic norms of conversational behavior across genres of dialogic interactions. What makes it an ideal choice for our efforts is the historical fact that it grew up side-by-side with one of the few sociological theories that was informed by linguistic data. As such, in contrast to other existing linguistic theories, it was fashioned specifically for the purpose of explaining sociological processes at multiple levels and within a wide range of contexts. Beyond these social phenomena, mechanisms such as grammatical metaphor (Halliday, 1994) endow it with the ability to represent how reasoning processes and conceptual development are displayed through language as well. Thus, it holds the potential to bridge between more cognitively oriented theoretical perspectives from the Learning Sciences as well as more socially oriented ones. We will introduce three constructs from Systemic Functional Linguistics that we believe are valuable for analysis of collaboration.

In the remainder of the chapter we will first discuss the role of conversation in collaborative learning. We will then discuss how conversational processes can be operationalized using constructs from the Learning Sciences as well as Linguistics, with an emphasis on Systemic Functional Linguistics. We will then integrate these perspectives and then discuss work towards automation of these constructs using machine learning and text mining technology. Finally, we discuss limitations of this work as well as future work.

\section{The Role of Conversation in Collaborative Learning}

The importance of linguistic analysis of collaborative learning is directly related to the importance places on conversational interactions in collaborative learning encounters. Beyond 
that attribution, we must also understand how we come to know and evaluate the role of those interactions, which are insights we draw from research methodology and the philosophy of science. Here we summarize our conceptualization of the lay of the land, which draws from previously published comparisons across research methodologies (Morrow \& Brown, 1994). We will describe the role that linguistic analysis has played along this continuum, showing how linguistic analysis may play a bridge-building role between alternative methodological and theoretical camps within the learning sciences.

Before we can begin to ask what the role of linguistic communication is in group learning we must consider what we mean by learning. At one end of the spectrum, within the Cognitivist tradition, the most common view is that learning is cognitive restructuring, which is typically studied with the individual student as the unit of analysis. At the extreme end within this paradigm, the role of discussion is mainly viewed as a stimulus for triggering cognitive processes, which are seen as the more direct causes of learning. At this endpoint, research questions related to collaborative discussion tend to focus on evaluating conversational patterns with respect to which cognitive processes they trigger and why, as well as how they rank in terms of their ability to trigger those processes. Secondary questions focus on how to increase the concentration of these valuable processes so that more opportunities to learn will be provided. In order to understand conversation in this light, conversational patterns must be categorized and defined in a reproducible way so that they can be counted. Otherwise it is not possible to determine whether there is a statistical relationship between that occurrence and learning. It is not surprising that within this sphere, typical approaches to analyzing collaborative discussions have fallen into what is known as "coding and counting". Within the Cognitivist tradition, although the main purpose of conversational interactions may be as a 
stimulus for learning, the social function of language is still acknowledged. For example, the motivational benefits of social interaction in learning contexts are often considered as benefits of collaborative learning even within that paradigm. Sometimes socially oriented constructs, such as motivation, identification, or positive regard for peers are operationalized as outcome variables and related to conversational constructs through inferential statistics within that tradition.

Moving further along the continuum away from the Cognitivist paradigm, the idea of cognitive apprenticeship is one in which learning occurs through interaction in an apprenticeship-like fashion (Collins et al., 1989). In this case, like in the Cognitivist tradition, learning is located in the mind of the individual, and may still be measured with tests, but because it begins through interaction, conversational or otherwise, it is considered that learning is inherently social. Within this sphere, because the learning is a joint activity involving multiple actors, its conceptualization is subtly more complex than that of the Cognitivist tradition. It thus becomes less natural to justify a post-positivistic experimental approach, although not impossible. Losing post-positivism as a convenient methodology would render the researcher unable to make causal claims about what types of interactions improve learning. Thus, within this sphere we see two camps of researchers, sometimes at odds with one another, namely: those who hold to post-positivist practices for causal explanations that can serve as the foundation for interventions improving learning, and those who adopt a qualitative stance in order to preserve the complexity of the relationships between the participants within the joint action. Within the post-positivist camp, the need for "coding and counting" approaches to enable statistical inference pushes for the codification of the conversational processes, such as scaffolding and help exchange, among others, that are used within the apprenticeship process. On the other side, 
within the qualitative camp, more of an emphasis is placed on thick description for explaining socialization processes. Categories of behaviors may or may not be identified and labeled, and when they are, they may or may not be counted, since counting plays less of a role where the identification of causal connections is not the goal. Within the qualitative methodological sphere, Systemic Functional Linguistics style analyses have sometimes played a role in the process of developing thick descriptions (e.g., Iedema et al., 2003).

Taking a further step away from the Cognitivist tradition, in the Sociocultural tradition, learning is apprenticeship into deeper participation (Lave, 1993). The focus may still be on the individual, but the target is the individual's role within the community. Students learn through participation, first in a peripheral role, and then in more and more integral roles. As they participate, they observe the contributions of central community members in their more integral participation firsthand. The conversation between the student and community members through the apprenticeship process is key.

As we move still further along the methodological spectrum, the focus becomes more generally on socialization into community practices through conversation, and broadens from the individual within a community to the community's common practices. In this perspective, the focus is on cultural practices, or mediations (Morrow \& Brown, 1994), and how they are appropriated in interactions within a community. Within this sphere, conversation is not simply viewed as a means of scaffolding as it is within the Vygotskian apprenticeship model. But the cultural practices themselves become an object of study as well as how they are transmitted, mutated, and transferred from one context into the next. For example, Christie (1999) explains the connection between the field of Systemic Functional Linguistics and Bernstein's sociological theory, where analyses of linguistic style are used as markers of socialization processes within 
the home, the school, and other spheres, in order to investigate why students of working class families are more prone to fail in school. It is important to note that where the transfer of cultural practices from one community to the next is an important target of investigation, the object of study is an open system, and thus the research questions lend themselves to a qualitative approach much more readily. While Systemic Functional Linguistics provides detailed codifications of cultural practices through systems of choices speakers make in how they present themselves and their ideas within a social context, these cultural practices are viewed as resources that may be used creatively, viewed subjectively, and negotiated in the moment of interaction between the parties present (Bucholtz \& Hall, 2003; Iedema at el., 2003), thus again we see a move away from "coding and counting" as the primary approach to analysis of verbal data. Investigation of conversation from a linguistic perspective is the topic for the next section.

\section{Desiderata for a Linguistic Analysis Framework}

Here we will begin with a construct that has arisen within the cognitive learning tradition, but when we poke closer, we see how it has both cognitive and social associations. Earlier we discussed the idea that one function conversation plays in collaborative learning interactions is knowledge sharing, knowledge integration, and even knowledge creation. A property of collaborative discussions that is associated with productive group knowledge construction that has its roots within a Piagetian theoretical framework is Transactivity (Berkowitz \& Gibbs, 1997; de Lisi \& Golbeck, 1999). In order for a contribution to count as transactive, it must include a display of reasoning, and must in some way reference a previously articulated idea from the conversation. Sionti et al. (in press) describe a simple two dimensional operationalization of this construct in which one dimension signifies displays of reasoning processes, and a second 
dimension distinguishes reasoning displays that express a connection with a prior reasoning display from those that are merely externalizations. In our own prior work (Joshi \& Rosé, 2007) as well as that of others (Azimitia \& Montgomery, 1993; Weinberger \& Fischer, 2006) we see evidence that prevalence of Transactivity in collaborative discussions correlates with learning. Results from other synergistic work also raise questions about how social processes affect properties of conversation that lead to a cognitive effect. In the Azmitia \& Mongomery (1993) study, $5^{\text {th }}$ grade students worked in pairs on math problems. Half of the pairs (i.e., the experimental condition) were students who self-reported themselves as friends prior to the study. The other half (i.e., the control condition) were not friends prior to the study. Students who worked with a friend were found to be more successful in problem solving, at least on the most difficult problems, than students in the control condition. What explained this difference was the occurrence of transactive contributions in the discussion, which were more frequent in the experimental condition, and mediated the effect of friendship on learning. Here we see two important considerations. First, the status of the relationship between students appears to have an effect on the level of Transactivity. It could then be seen as a reflection of that relationship, and it would therefore be a mistake to relegate Transactivity as strictly within the purview of a Cognitivist theoretical framework. Second, the level of difficulty of the problem was also a factor in whether the relationship between friendship or Transactivity was significant or not. Therefore, we have more evidence that properties of conversational style, such as Transactivity, should be considered in connection with the content of what is being discussed. So, we are left with questions about the process through which friendship increases Transactivity in interactions, which is beyond the scope of what can be addressed within a purely Cognitivist framework, and yet is a question that should be important to that community based on this result. Furthermore, 
we can ask about the social processes related to friendship that affect the navigation within the discussion of the conceptual space and problem solving space of the problem being solved.

Other constructs associated with a Cognitivist perspective on learning also have social connections. We mentioned that in our earlier work (Joshi \& Rosé, 2007) we observed a significant correlation between the occurrence of Transactivity in collaborative design discussions in the thermodynamics domain and learning gains as measured by a test related to concepts in thermodynamics and Rankine cycles (Kumar et al., 2007). However, from an earlier study of individual learning supported by human tutors using the same design task (Rosé et al., 2006), the predictive value of a measure of topic coverage within a conversation is also significant and even more substantial. Thus, we must consider that it is both the content and how the content is discussed that explains how beneficial an interaction is for learning. Both of these can be considered within the purview of a purely Cognitivist framework. However, we are left wondering what factors, possibly social, affected which concepts were discussed and how?

In another example, Wang and colleagues (2007) present a study that argues for considering a connection between cognitive factors and social factors, even when characterizing learning within a Cognitivist framework. This study presents a $2 \mathrm{X} 2$ factorial design where students worked either individually or in pairs, either with feedback or without feedback, in an idea generation task. Consistent with earlier work on process losses in group idea generation (Dugosh et al., 2000; Nijstad \& Stroebe, 2006), students who worked in pairs produced significantly fewer ideas than those in the condition where students worked individually. A posthoc analysis of the conversational interactions supported the interpretation that the process losses were an effect of cognitive interference between students (Wang \& Rosé, 2007a). While this is not surprising, what is surprising is that students in the pairs condition also learned significantly 
less, and that the learning was mediated by success at the idea generation task. The feedback was designed to mitigate the process loss effect without offering any domain level instruction, using a type of idea generation support discussed in earlier work (Dugosh et al., 2000). Pairs with feedback produced significantly more ideas and learned significantly more than pairs without feedback. In additional posthoc analyses of the conversational data, pairs with feedback stayed on topic longer and talked more similarly to one another than pairs without feedback (Wang \& Rosé, 2007b). Thus, the feedback, designed to achieve a cognitive effect, also had a social effect, as displayed through linguistic properties of the conversation. While these characteristics of conversation are not formally the same as Transactivity, what we see here is related. Students were informally observed to refer to each other's ideas and build off of each other's ideas frequently in the feedback condition. The overall picture is that the content of the discussion was what was demonstrated to have a strong predictive relationship with learning gains. And yet, social factors influenced the extent to which those cognitive factors were present. The introduction of feedback did not completely mitigate the process loss effect, however, it lead both to significantly higher learning gains and a richer interaction. Shallow indicators of conversational richness (i.e., staying on the same topic longer and demonstrating higher lexical cohesion between speakers) also significantly predicted learning separately from content oriented concerns. However, just as the predictive value of Transactivity alone was low in the Joshi \& Rosé (2007) study, the predictive value was substantially lower than that of the number of ideas discussed within the conversation. Nevertheless, a deeper look at the social interaction might allow us to understand how the feedback affected the dynamic between students and thereby influenced the direction the conversation took, which then influenced the learning. 


\section{Integrating Cognitive and Social Perspectives through Systemic Functional Linguistics}

Now that we have introduced a variety of views on why conversation is believed to be important for collaborative learning, and important for what, we can now dig deeper into what conversation is and what makes it work, especially with respect to integrating theoretical perspectives.

The field of Systemic Functional Linguistics provides a wealth of constructs that can be used as lenses through which to view the stylistic choices of conversational participants. However, in order to bound our work for the purpose of this chapter, we limit ourselves to three, namely Martin \& Rose's (2007) Negotiation system, Martin \& White's (2005) conceptualization of Bakhtin's notion of heteroglossia (Bakhtin, 1986), and the more elaborate Engagement system, which is part of the larger system of Appraisal.

We illustrate the operationalization of our chosen constructs using example interactions that come from the thermodynamics domain where sophomore mechanical engineering undergrads are working together in pairs to design a power plant using a paradigm known in mechanical engineering as the Rankine cycle. We have run a series of studies investigating learning in this design task over the past several years, both in connection with individual learning (Rosé et al., 2005), collaborative learning (Chaudhuri et al., 2009; Ai et al., 2010), and the comparison between the two (Kumar et al., 2007). In recent years (Chaudhuri et al., 2009; Ai et al., 2010) we have assigned the two students in each pair to opposing goal conditions.

Specifically, one student was assigned the goal of achieving the highest power output as possible (Power condition), and the other student was assigned the goal of achieving the lowest level of negative environmental impact (Green condition). The students were told that they had to work together to agree on a single design, but that they would be evaluated individually with respect to 
how well their group design met their personal design objective. Nevertheless, the instructional objective of the activity is not to produce a good design but for students to explore the design space and understand the implications of design choices in light of the theory. Thus, the apparently opposing goal orientations are simply to provide a motivation for the exploration of the design space.

\section{Negotiation}

What we are concerned with in the Negotiation system is the codification of how information, goods, and services are exchanged within a conversation.

\section{Defining Negotiation}

In a collaborative learning setting, relevant goods and services frequently include helping actions. However, the Negotiation system is more general than this, and can be applied to the exchange of all types of goods and services through conversation. For example, something as different from help exchange as a clerk requesting a customer to pay a certain amount of money can be analyzed within the same framework. What makes this system particularly valuable is the way it serves to provide a natural segmentation of an ongoing interaction into episodes where some transaction between parties within the conversation has been accomplished, i.e., either some piece of information has been exchanged, or some service has been rendered. These small accomplishments then become the building blocks for larger and more complex accomplishments that might require a more concerted, long term effort, such as building an integrated understanding of a phenomenon, developing a plan, or solving a problem. Some of these exchanges may occur with minimal representation in terms of conversational moves. For 
example, a speaker could simply express an unsolicited piece of information, and it could be tacitly accepted without comment by the other members of the conversation. In this case, the exchange may require only one conversational move. However, it may not be that simple. The full exchange system allows for exchanges to transpire over multiple moves, which can all be seen as connected. The provision of the information or goods and services, and possibly the preceding request if there was one, are treated as being in a prominent position within the exchange. Other kinds of moves play a supporting role in the exchange.

Within the Negotiation framework, speakers take up transitory speech roles within an exchange structure, in which one speaker takes up the primary role, thereby placing the other speaker into the secondary role. It can also happen that a speaker places himself into a secondary role, which then casts the other speaker into a primary role. In group discussions, overhearers who are present but are neither cast in the primary or secondary role are by default cast into a tertiary role. Where information is exchanged, the roles are termed primary knower and secondary knower. Where goods and services are exchanged, the roles are termed primary actor and secondary actor. For example, when a clerk requests a customer to pay, that clerk is placing himself within the secondary actor role, and thus casting the customer in the primary actor role, in this case, the one who is the source of the payment.

\begin{tabular}{|l|l|l|}
\hline & & Negotiation \\
\hline Student 1 & I don't know what to do. & Secondary Knower \\
\hline $\begin{array}{l}\text { Student } 2 \\
\text { Student } 1\end{array}$ & What does it say on the sheet? & setup move \\
\hline $\begin{array}{l}\text { Student } 2 \\
\text { Student1 }\end{array}$ Right. & any idea what we open to start? & Secondary Knower \\
\hline $\begin{array}{l}\text { Student2 } \\
\text { I just opened the reheat cycle. It's blank, and it's already a system. We can } \\
\text { substitute values in as we go. }\end{array}$ & Primary Knower \\
\hline Student1 & $\begin{array}{l}\text { hmokay, do you understand how to maximize the efficiency of a reheat } \\
\text { cycle? }\end{array}$ & Primary Knower \\
\hline
\end{tabular}

Figure 1 Chat examples coded for Martin \& Roses's (2007) Negotiation Framework. 
While implications with respect to status relationships can be drawn from speech roles where they are treated as sanctioned, such as the teacher's role as primary knower within an Initiation-Response-Evaluation (IRE) classroom (Veel, 1999), we will see that this need not be the case. Typically the roles of primary and secondary speakers are highly volatile and do not appear to carry any particular lasting significance with respect to status distribution within the conversation. Frequent shifting in speech roles may even serve to underscore the equal footing between the two students, possibly despite their difference in ability level. Furthermore, speech roles are meaningful even where transitory in that they signify which speakers are treated as the source and recipient respectively of what is being exchanged. Thus, it allows us to ask not only which speakers are cast as authoritative within an interaction, but authoritative with respect to what.

\section{Applying Negotiation}

Now we offer an example where we can see the value of examining collaboration from a Systemic Functional Linguistics perspective. This is a reanalysis of a dataset from a previously published study (Kumar et al., 2007b). Thirty sixth-grade students worked in pairs on fractions word problems in two conditions: a personalization condition, and a control condition. The pairs of students communicated through a computer interface that was part chat window (like an instant messenger) and part intelligent tutoring system. The first day had a lab session, then a pretest and the second day had another lab session and a posttest quiz as well as collaboration questionnaire. From the previously published analysis of the data there was a significant increase in perception of amount of help given and received, and there were also marginally higher learning gains for the personalization condition $(\mathrm{p}=.06$, effect size $.55 \sigma)$. There was also 
a significant increase in amount of help given per problem $(F(1,15)=16.8, p<.001$, effect size 1 $\sigma)$, as well as students being marginally more likely to complete a step on their own after receiving help $(\mathrm{p}=.07)$ (Cui et al., 2008). An informal analysis of the discussion data also noted that there were many more insults exchanged between students in the Control condition. So we see strong trends that indicate both more learning and more of a tendency to complete a step on their own after experiencing difficulty. This would make considerable sense as a connection within a Cognitivist perspective. However, what that perspective does not tell us is why there was more of a tendency to complete a step after having difficulty in the personalization condition, and what the connection was with the negative affective behavior, if any. Our reanalysis suggests an answer.

In the reanalysis we recoded for authoritative behavior using the Negotiation Framework, giving each student an authoritativeness score for each lab day based upon the proportion of primary knower statements to total number of primary and secondary knower statements. Each student also received a shift score, which was computed from the residual after doing a simple linear regression predicting day 2 authoritativeness from day 1 authoritativeness. We also used a naïve coding of "aggressiveness" to identify insults and exhibits of pushy behavior. The student in each pair who was observed to engage in more instances of aggressive behavior was labeled with a binary indicator as the Aggressor, whereas the other student was labeled as the de facto Victim.

The simple personalization intervention resulted in significantly less aggressiveness $(\mathrm{F}(1,56)=8.93, \mathrm{p}<.005$, effect size $.63 \sigma)$. There was significantly more aggressiveness on day 2 $(\mathrm{F}(1,56)=15.61, \mathrm{p}<.0005$, effect size $.87 \sigma)$. However, there was a significant interaction with condition $(\mathrm{F}(1,56)=6.06, \mathrm{p}<.05)$, which showed that the only increase in aggressiveness on day 
2 was in the control condition. Only in the control condition on day 2 did the identified aggressors exhibit significantly more authoritativeness than targets of the aggression $(F(1,23)=$ $5.92, \mathrm{p}<.05)$. Also, only in the control condition did aggressors become significantly more authoritative than predicted for day 2 , and control condition victims become significantly less authoritative than predicted, $(\mathrm{F}(1,23)=5.28, \mathrm{p}<.05$, effect size $.15 \sigma)$.

We would expect instances of aggressiveness and changes in authoritativeness to have an effect on learning, as hostile learning environments are not conducive to positive learning gains. However, looking at the learning analysis, we do not see a main effect of aggressiveness on learning, but with a post-hoc analysis we see that victimized students in the control condition learned significantly less than the aggressors as well as less than all students in the personalization condition $(\mathrm{F}(1,18)=9.26, \mathrm{p}<.01)$. This is possibly due to how help was received differently in the control condition. Shifting to a less authoritative stance than predicted reduced learning in the control condition, and there was a significant correlation between shift and learning only within that condition $(\mathrm{F}(1,20)=7.91, \mathrm{p}=.01)$. In the personalization condition there was barely any variance in shift, which might explain the lack of correlation.

In each of the dimensions analyzed, the results favor the personalization condition. We see that the control condition in this study appears to have created a social environment in which the students who portrayed more aggressive behavior on the first day, shifted to a more authoritative stance than predicted while the targets of the aggression shifted to a less authoritative stance than their first day scores predicted. We also see that students in that condition, when they experienced trouble with a step, tended to drop out of the picture and let their partner finish the step for them. Meanwhile, both aggressors and victims in the personalization condition barely shifted more/less in authoritativeness. And, consistent with this, 
students had more of a tendency to continue trying in the face of difficulty. We see here then, through this linguistic analysis, a potential social explanation for a behavior that lead to a cognitive effect.

\section{Heteroglossia and Engagement}

As a second construct that we consider, the Martin \& White (2005) notion of heteroglossia yields further insights into the status relationship between the two speakers. A third but closely related construct, the Engagement system, begins with the distinction between heteroglossia and monoglossia, but expands this notion with a system of finer and finer grained distinctions.

\section{Defining Heterglossia and Engagement}

Note that the general notion of monoglossia versus heterglossia from Bakhtin seems to be much broader than the notion referred to in Martin and White. Heteroglossia always refers to multiple voices in a text or in a discourse community, but it can mean, for example, different registers in which someone speaks depending upon what context they are in. In Martin and White (2005), heteroglossia refers to ways in which speakers directly encode in their expression of a clause their awareness of other stakeholders in the interaction.

In Martin and White's specification of what counts as heteroglossic as a precursor to analysis using the Engagement system, which we will discuss next, three requirements must be met: first, some propositional content must be asserted in some form, although it may be done in such a way as to communicate extreme uncertainty. Thus, questions that are framed where the speaker was asking an honest question, and not expecting a specific answer do not count as heteroglossic. Interjections, like "Yay", that cannot be interpreted as ellipsis, and thus have no 
propositional content are not considered heteroglossic, however, fixed expressions like "no", and "yes" that implicitly assert the propositional content of the yes/no question they are a response to do count as expressing propositional content. Other forms of ellipsis (e.g., "coal" in response to "Which type of fuel would you choose?") and do-anaphora (i.e., "I did." In response to "Did you select a fuel type?") also count as having propositional content. Second, an awareness must be made visible to the presence of alternative perspectives than that represented by the propositional content of an utterance. Thus, bald claims, even if they are biased, do not acknowledge alternative perspectives. For example, "Natural gas is the obviously superior choice" is undoubtedly subjective, but it is not heteroglossic. It does not show any awareness that someone else might disagree. A third requirement is that in order to count as heteroglossic, the acknowledgement of other perspectives must be expressed grammatically (e.g., through a model auxiliary like "might") or paraphrastically (e.g., "I think") within the articulation of that propositional content rather than signaled only through the discourse structure. Figure 2 presents an illustrative example.

\begin{tabular}{|l|l|l|}
\hline Student1 & $\begin{array}{l}\text { hm okay, do you understand how to maximize the efficiency of a reheat } \\
\text { cycle? }\end{array}$ & No Assertion \\
\hline Student2 & $\begin{array}{l}\text { I think I do, but since we have opposing goals i'll be helping you along to a } \\
\text { point. =) } \\
\text { Anyway, we have constants that we have to deal with such as the } \\
\text { material being SS and the fluid being water. }\end{array}$ & Heteroglossic-Expand \\
\hline $\begin{array}{l}\text { Student1 } \\
\text { Student2 }\end{array}$ & $\begin{array}{l}\text { No, no, I'm going for green. Yay team. } \\
\text { Anyway, did you want to do reheat or simple? You seemed to have } \\
\text { reservations before. }\end{array}$ & Monoglossic \\
\hline $\begin{array}{l}\text { Student1 } \\
\text { i think reheat. I'll explore the simple one quickly. }\end{array}$ & Heteroglossic-Expand \\
\hline Student2 & By the way, I take it your goal is power? & Heteroglossic-Expand \\
\hline $\begin{array}{l}\text { Student1 } \\
\text { ytudent2 }\end{array}$ & $\begin{array}{l}\text { Great... Well, the biggest variable is fuel then, not so much reheat or } \\
\text { simple. }\end{array}$ & Heteroglossic-Expand \\
\hline Well, I would like to do solar, what about you? & Monoglossic \\
\hline
\end{tabular}

Figure 2 Examples of Martin \& White's Heteroglossia construct. 
The construct of heteroglossia introduces the notion that the voice of the speaker is situated among other voices. But beyond that acknowledgement of the existence of other voices, what we do not see in this simple binary distinction is the manner of that positioning. The details of that positioning are further specified within Martin and White's Appraisal framework, which includes Attitude, in which feelings are revealed towards propositional content, Graduation, in which feelings are either magnified or downplayed, and Engagement, in which a speaker positions herself in relation to the propositional content of the utterance, positions the audience in relation to the propositional content, and positions herself in relation to the audience (Martin \& White, 2005). Thus, it gives us the ability to identify how the choices speakers and writers make position that person within their speech context in relation to the other conversational participants as well as with the content of the discussion.

Once we have determined that an utterance counts as heteroglossic, we can then subdivide it into utterances that Contract the positions or perspectives treated as viable within a conversation, or conversely, ones that Expand the scope of what is treated as viable. Either way, an acknowledgement is made that more than one way of looking at the world is at play.

Utterances that contract that scope, such as making an absolute assertion that leaves no room for questioning, or outright rejecting a position, are typically seen as taking a more authoritative stance than ones that expand the options, such as making a suggestion. This notion of levels of authoritativeness is one important component of expressing the positioning of the speaker in relation to the propositional content. However, it also says something about where the speaker positions himself in relation to the audience. Taking an authoritative stance casts the other speaker into a less authoritative stance. However, when this system is further subdivided, we see other options for positioning. For example, a Distancing move, in which the source of authority 
is ascribed to a third party, allows an authoritative statement to be made, which may contract options, but does not interfere with the positioning between the speaker and the audience. The speaker remains committed to the authoritative proposition, but not responsible for it. For example, if a student says "The book says solar power is a good choice.", then the student may be presenting information that he knows the other student will be opposed to, but he positions the book as the party standing in opposition, rather than himself.

\section{Applying Heterglossia and Engagement}

Now we will discuss the connection between Transactivity from the Collaborative Learning community and the related constructs of Heteroglossia and Engagement from the Systemic Functional Linguistics community. As earlier, we illustrate this with an example interaction from the thermodynamics domain.

In the collaborative discussion presented in Figure 3, Student 1 has been assigned to the Power condition, and Student 2 has been assigned to the Green condition. They discuss their opposing goal orientations in the first nine contributions, and in the remainder of this excerpt, they discuss which type of fuel they should use in their Rankine cycle, examining their choices based on implications for these two opposing goals. In the end, they settle on solar-thermal, which is a good choice for Green, but not Power. Thus, one could consider that on this aspect of the design, Student 2 has achieved an advantage in terms of how the design will be evaluated in light of the two opposing goal orientations.

Transactive conversational contributions are viewed as important steps in a knowledge building or consensus building process (Weinberger \& Fischer, 2006). In making connections between newly articulated ideas and material contributed earlier in a conversation, ideas build 
upon one another, and differing understandings are elaborated, integrated, and possibly

transformed. In the example presented in Figure 3, we see the decision making process about the selection of fuel unfold. Key moves from a decision making standpoint include where they agree to find a decision that would be a compromise between their two opposing goal orientations, where the idea of encoding the fuel choice as a number is articulated, which then becomes a theme carried throughout the remainder of the conversation, and where the final choice is introduced. All three of these are coded as transactive.

\begin{tabular}{|c|c|c|c|}
\hline & & Transactive & Engagement \\
\hline Student1 & any idea what we open to start? & Not Reasoning & No Assertion \\
\hline Student2 & $\begin{array}{l}\text { I just opened the reheat cycle. It's blank, and it's already a system. We can substitute } \\
\text { values in as we go. }\end{array}$ & Not Reasoning & Monoglossic \\
\hline Student1 & hm okay, do you understand how to maximize the efficiency of a reheat cycle? & Not Reasoning & No Assertion \\
\hline \multirow[t]{2}{*}{ Student2 } & I think I do, but since we have opposing goals i'll be helping you along to a point. =) & Externalization & Heteroglossic-Expand \\
\hline & $\begin{array}{l}\text { Anyway, we have constants that we have to deal with such as the material being SS } \\
\text { and the fluid being water. }\end{array}$ & Not Reasoning & Monoglossic \\
\hline Student1 & woah woah woah, what's your goal? Efficiency? & Not Reasoning & Heteroglossic-Expand \\
\hline \multirow[t]{2}{*}{ Student2 } & No, no, I'm going for green. Yay team. & Not Reasoning & Monoglossic \\
\hline & $\begin{array}{l}\text { Anyway, did you want to do reheat or simple? You seemed to have reservations } \\
\text { before. }\end{array}$ & Not Reasoning & Heteroglossic-Expand \\
\hline Student1 & i think reheat. I'll explore the simple one quickly. & Not Reasoning & Heteroglossic-Expand \\
\hline Student2 & By the way, I take it your goal is power? & Not Reasoning & Heteroglossic-Expand \\
\hline Student1 & yup & Not Reasoning & Monoglossic \\
\hline \multirow[t]{2}{*}{ Student2 } & Great... Well, the biggest variable is fuel then, not so much reheat or simple. & Transactive & Heteroglossic-Contract \\
\hline & Well, I would like to do solar, what about you? & Not Reasoning & Heteroglossic-Expand \\
\hline \multirow[t]{2}{*}{ Student1 } & ha i'd try natural gas for power. but i dont understand this very well. & Externalization & Heteroglossic-Expand \\
\hline & $\begin{array}{l}\text { do we need to come up with the same system? if so, we'll have to do something in- } \\
\text { between }\end{array}$ & Transactive & Heteroglossic-Expand \\
\hline \multirow[t]{2}{*}{ Student2 } & Yes, we need to agree on a system that fits both of our goals. & Not Reasoning & Monoglossic \\
\hline & $\begin{array}{l}\text { Well, single cycle is more friendly to the environment, so If we do that I'll conced to } \\
\text { nuclear as the fuel. }\end{array}$ & Transactive & Heteroglossic-Expand \\
\hline Student1 & $\begin{array}{l}\text { ok let's do single cycle and go with.... coal? nuclear isnt allowed:( natural gas is } 2560 \text {, } \\
\text { coal is } 2400 \text {, oil is } 800 \text {, etc }\end{array}$ & Transactive & Heteroglossic-Expand \\
\hline Student2 & $\begin{array}{l}\text { Nuclear isn't allowed in the program, but it still can be used by manually entering the } \\
\text { Q value. }\end{array}$ & Transactive & Monoglossic \\
\hline Student1 & True. eh lets just go with coal. so lets do 2400 & Externalization & Heteroglossic-Expand \\
\hline Student2 & Coal, the worst of the fuels for green. & Transactive & Monoglossic \\
\hline Student1 & kk want to do solar-thermal? 240 ? Your end goal is just "green"? & Transactive & Heteroglossic-Expand \\
\hline Student2 & I'd love to, do you want to use it? Yep, green at any cost. & Not Reasoning & Heteroglossic-Expand \\
\hline Student1 & ya lets go with it & Not Reasoning & Heteroglossic-Expand \\
\hline
\end{tabular}

Figure 3 A collaborative design exercise coded for Transactivity and Heteroglossia.

In this interaction, we see both students articulating their reasoning in a transactive manner throughout the discussion, which might suggest an equal status relationship between the 
speakers, based on Piaget's theory. However, more of those were contributed by Student 2, which might suggest an imbalance. At least Speaker 2 exhibits a greater ability to articulate reasoning and to relate those reasoning displays to previously articulated material. A Heteroglossic contribution need not be counted as an explicit display of reasoning. Thus, it would not serve the function of assessing the ability to articulate reasoning the way Transactivity may, although there are other constructs like grammatical metaphor in systemic functional linguistics that have been used to assess level of sophistication of thought displayed through writing (Halliday, 1994).

Because of being applicable to contributions where reasoning is not explicitly displayed, what Heteroglossia gives us beyond what is marked in transactive utterances is a more exhaustive accounting of how the power balance is maintained through a conversation. Throughout the conversation, we see both speakers doing work to maintain their solidarity, often through heteroglossic contributions that express deference to the other student's perspective. For example, "We have constraints that we have to deal with" casts the obligations towards the task at hand and their opposing goal orientations as coming from outside the interaction, and thus reduces the importance of internal pressures. "Did you want to do reheat or simple?" offers two alternatives without expressing preference, and thus leaves both options open, rather than restricting to a personal preference. The other speaker's response "I think reheat" expresses a preference, but not in a way that limits options. We know from prior work that heterogeneous learning groups can be problematic, and inequalities within the status balance can easily ensue, which interfere with the productive functioning of the group. Heteroglossia allows us to see students working to avoid falling into this behavior. It may eventually shed light on what influences which topics students spend time considering within an interaction. 


\section{Technology for Automatic Conversation Analysis}

Earlier in the chapter, we highlighted different aspects of collaborative discussions that are relevant depending upon the theoretical and methodological framework that is used as a lens through which collaboration is studied. In particular, we have highlighted the potential value that linguistic analysis, such as those related to constructs we have discussed from Systemic Functional Linguistics, may be of value to the CSCL community. In this section we focus on how we can develop computational models that can assist in automating such analyses. We will begin this discussion with a description of work which has been done on problems similar to those we would like to study, introducing concepts from machine learning as they are necessary. Then, we conclude with a preliminary view of how to build computational models of Systemic Functional Linguistics constructs using machine learning technology.

\section{Looking Back: A historical perspective on text mining}

In recent years, the computer supported collaborative learning community has shown interest in automatic analysis of data from collaborative learning settings, building on and extending work in text mining from the language technologies community. Application of such technology can be very direct, particularly where interests are very similar, such as detecting where attitudes are encoded in the expression of ideas, which is currently a very active area of research in the language technologies community. In other cases, the specific types of analysis are less directly related to what is most centrally of concern to the language technologies community, for example, the operationalizations of knowledge building and social positioning. While there is a body of work in collaborative learning - for instance, studying automatic analysis of 
Transactivity (Ai et al, 2010; Rosé et al, 2008; Joshi \& Rosé, 2007) and other collaborative learning constructs (McLaren et al, 2007; Wang \& Rosé, 2007) - these are the exception.

Automatic analysis approaches as we know them today are only capable of identifying patterns that occur in a stable and recognizable way. Although those patterns can be arbitrarily complex, there are limitations to contexts in which an approach of this nature is appropriate. These approaches are most naturally usable within research traditions that value abstraction and quantification. The most natural application of such technology is within traditions that employ coding and counting approaches to analysis of verbal data. Nevertheless, recent work pushes beyond these strict boundaries. For example, Ai and colleagues (2010) have demonstrated how a structured topic modeling approach can be used to measure influence within a conversation between one conversational participant and another in a graded way, which was validated through questionnaire data. Nevertheless, the very idea of operationalizing collaboration practices in a rule-based way, even if those rules can be arbitrarily complex functions, requires some abstraction and simplification of reality. Thus, research traditions that value preservation of complexity and avoid abstraction and generalization will find it uncomfortable to consider using such an approach in their research. By nature, empirical modeling approaches involving statistics and machine learning are mainly useful for capturing what is typical. In contrast, within many qualitative research traditions, it is the unusual occurrences and practices that are worthy of study. Thus, it is unlikely that such technology would be directly usable for producing the kind of findings that are valued within those traditions. However, what it may be able to assist with is finding the unusual occurrences within a mass of data, which might then be worthy of study in a more traditional way. 
The wide range of conversational constructs that have been explored within this chapter provide new opportunities for exploration of automatic analysis technology and ways in which that technology can be improved. Machine learning algorithms are designed with the goal of finding mappings between input features and output categories. They do this by collecting handcoded "training examples" for each output category, then using statistical techniques to find characteristics that exemplify each category. The goal of such an algorithm is to learn general rules from these examples, which can then be applied effectively to new data. In order for this to work well, the set of input features must be sufficiently expressive, and the training examples must be representative. Machine learning researchers are tasked with designing input features expressive enough to capture the subtleties of the categories they are trying to learn. At the shallowest level, these input features are simply the words in a document. Many other features, such as word collocations, part of speech tags, and low-level lexical features are routinely used.

In the past decade, a variety of approaches to text categorization have been developed and refined. Major advances have been made due to the development of powerful machine learning algorithms, the increase in speed of computers, and the availability of very large relevant corpora. The biggest limitation, perhaps, is the shallow nature of the representations of text that are typically used in this computational work, which treat text as though it were an unstructured collection of words, or pairs of words, or pairs of grammatical categories. We have made this argument in earlier publications related to analysis of attitudes displayed in text (Joshi and Rosé, 2009). These two problems - the features used to represent the text to be analyzed, and the annotation of the text in a way that suitably operationalizes the construct of interest - are two sides of the same coin. The operationalization determines which instances should be grouped and distinguished from instances in other groups. And the features used to represent the text 
allow the definition of the conditions under which instances are assigned to groups to be expressed. Unless these two are in alignment, the model building will not be successful no matter how powerful of a machine learning algorithm and how much training data is available. Thus, both of these issues are vital to future progress in computational linguistics.

Even in much earlier work, limitations of feature spaces have been noted in connection with topic and content oriented classification tasks such as home page classification (Furnkrantz et al., 1998), and knowledge component classification for an essay grading task (Rosé \& VanLehn, 2005). Closer to the topic at hand, text representation was the key to improving performance of classification of segments of a newsgroup style discussion with a 5-category operationalization of Transactivity (Rosé et al., 2008). In that work, the key additional features that improved performance were ones that represented how similar a segment was to others contributed earlier by other participants in the discussion as well as features that represented the position of the segment within a message, and the position of the message on a thread.

What is important here from the standpoint of a CSCL research considering using such technology as part of his or her research methodology is to understand what kind of thinking and preparation is necessary in order to use machine learning to the best of its potential.

\section{Computational Model of Negotiation}

Currently, results on computationalization of Systemic Functional linguistics are somewhat limited. So far, to the best of our knowledge, there has not been any published work on the Engagement system or even the simpler notion of Heteroglossia. Argamon and colleagues have worked on a computationalization of a different construct referred to as Attitude (Whitelaw and Argamon, 2004; Argamon et. al, 2007), which is one of three constructs under the broader 
construct of Appraisal, of which Engagement is another one. The primary computational approach pursued in that work has been to build lists of cue words which are likely to occur only in certain contexts, such as "worst," "awful," and "terrible" with a negative attitude. Such a list of words is termed a "sentiment lexicon". The initial approaches to machine learning for Appraisal theory used lexicons like these. Initially, the lists contained only adjectives (Taboada and Grieves, 2004), but later work expanded this format to a complex taxonomy of words and cue phrases (Whitelaw and Argamon, 2004). This approach is popular because it requires no hand annotating of training examples (words in the lexicon can be found automatically); because it can be expanded to larger lists because the level of detail in the list can be adapted to individual problems and because such lexicons can be built automatically in some cases.

Our work has so far focused on the Negotiation system. We found, however, that a key phrase matching approach such as taken by Argamon and colleagues in connection with Attitude would not work for Negotiation. The same contribution uttered in different contexts can take on a totally different status within the Negotiation framework. Thus, we needed a framework that would allow us to leverage these contextual constraints in the interpretation process. In order to accomplish this, we have employed a machine learning paradigm referred to as Integer Linear Programming (ILP), which allows us to directly express the contextual constraints on sequencing and repetition that come from the definition of the codes within that construct. If we take a simpler approach where we make predictions about the codes just from the content of the contributions themselves, our machine generated authoritativeness measures explained only $35 \%$ of the variance in those computed based on human annotations, where inter-annotator agreement on the human codes has been evaluated at .71 Kappa. In contrast, the ILP formulation that leverages context is able to explain $95 \%$ of the variance in scores computed based on human 
annotations. Thus we see how important it is for the considerations that are built into the computational framework to reflect the conceptual essence of the definitions of those constructs.

\section{Limitations and Challenges}

We conclude this chapter by outlining the limitations of this review and discussion of the biggest challenges we seek to address moving forward. We started out the chapter outlining the major subcommunities within the learning sciences, how they view learning differently, and how those differences change the role they see discussion as playing in the learning. We have introduced some subcommunity-specific frameworks for investigating issues that are of concern within the associated communities. We raised questions that fall at the frontiers between communities and have argued how we think Systemic Functional Linguistics may have the potential to serve as an aid to communication between communities, as an extensive descriptive linguistic framework encompassing aspects of linguistic expression that are interesting when viewed by the full gamut of perspectives and may serve as a useful boundary object. Much work is left to do to flesh out and formalize what has been provided by the Systemic Functional Linguistics community so that it can be applied broadly. Ultimately, our hope is to be able to develop automatic analysis technology, and we have outlined the state-of-the-art in language technologies related to Negotiation and some constructs within the Appraisal system.

\section{Acknowledgement}

This research was funded by grants from the National Science Foundation, including NSF SBE0836012, DRL-0835426, and HCC-0803482. 


\section{References}

Ai, H., Kumar, R., Nguyen, D., Nagasunder, A., Rosé, C. P. (2010). Exploring the Effectiveness of Social Capabilities and Goal Alignment in Computer Supported Collaborative Learning, in Proceedings of Intelligent Tutoring Systems, pp 134-143, Springer.

Argamon, S., Whitelaw, C., Chase, P., Hota, S., Garg, N., and Levitan, S. (2007). Stylistic text classification using functional lexical features. Journal of the American Society for Information Science and Technology 58(6), pp 802-822.

Azmitia, M., \& Montgomery, R. (1993). Friendship, transactive dialogues, and the development of scientific reasoning. Social Development, 2(3), pp 202-221.

Bakhtin, M. (1986). Speech genres and other late essays. Vern W. McGee (Trans.), C. Emerson \& M. Holquist (Eds.). Austin: University of Texas Press.

Berkowitz, M., \& Gibbs, J. (1979). Unpublished manual for coding transactivity

Bucholtz, M. \& Hall, K. (2005). Identity and interaction: a sociocultural linguistic approach. Discourse Studies 7(4-5), pp 585-614.

Chaudhuri, S., Gupta, N., Smith, N. A., Rosé, C. P. (2009). Leveraging Structural Relations for Fluent Compressions at Multiple Compression Rates. Proceedings of the Association for Computational Linguistics, pp 101-104, Association for Computational Linguistics.

Christie, F. (1999). Pedagogy and the Shaping of Consciousness: Linguistic and Social Processes. London: Cassell

Collins, A., Brown, J.S., and Newman, S.E. (1989). "Cognitive Apprenticeship: Teaching the Craft of Reading, Writing and Mathematics" In L.B. Resnick (ed.) Knowing, Learning, and Instruction: Essays in Honor of Robert Glaser Hillsdale, NJ: Erlbaum 
Cui, Y., Chaudhuri, S., Kumar, R., Gweon, G., Rosé, C. P. (2009). Helping Agents in VMT, in G. Stahl (Ed.) Studying Virtual Math Teams: Computer Supported Collaborative Learning 11(4), pp 335-354, Springer.

de Lisi, R., \& Golbeck, S.L. (1999). Implications of the Piagetian Theory for peer learning. Cognitive perspectives on peer learning, pp. 3-37, Lawrence Earlbaum Associates.

Dugosh, K. L., Paulus, P. B., Roland, E. J., \& Yang, H. (2000). Cognitive stimulation in brainstorming. Journal of Personality and Social Psychology. 79(5), 722-735.

Furnkranz, J., Mitchell, T., Mitchell, M., \& Riloff, E. (1998). A Case Study in Using Linguistic Phrases for Text Categorization on the WWW. Proceedings from the AAAI/ICML Workshop on Learning for Text Categorization, pp. 5-12, AAAI Press.

Halliday, M. (1994). An Introduction to Functional Grammar. London: Edward Arnold

Iedema, R., Degeling, P.,Braithwaite, J., White, L. (2003). 'It's an Interesting Conversation I'm Hearing': The Doctor as Manager, Organizational Studies 25(1), pp 15-33.

Joshi, M. \& Rosé, C. P. (2007). Using Transactivity in Conversation Summarization in Educational Dialog. Proceedings of the SLaTE Workshop on Speech and Language Technology in Education, IEEE Signal Processing Society.

Joshi, M. \& Rosé, C. P. (2009). Generalizing Dependency Features for Opinion Mining, Proceedings of the ACL-IJCNLP 2009 Conference Short Papers, pp 313-317.

Kumar, R., Rosé, C. P., Wang, Y. C., Joshi, M., Robinson, A. (2007). Tutorial Dialogue as Adaptive Collaborative Learning Support, Proceedings of Artificial Intelligence in Education, pp 383-393, IOS Press. 
Kumar, R., Gweon, G., Joshi, M., Cui, Y., Rosé, C. P. (2007b). Supporting Students Working Together on Math with Social Dialogue. Proceedings of the SLaTE Workshop on Speech and Language Technology in Education, IEEE Signal Processing Society.

Lave J. (1993) The practice of learning. In S. Chaiklin \& J. Lave (Eds.), Understanding Practice: Perspectives on Activity and Contex, Cambridge: CUP.

Martin, J. R., \& Rose, D. (2007). Working with Discourse: Meaning Beyond the Clause. Continuum

Martin, J. R., \& White, P. R. (2005). The Language of Evaluation: Appraisal in English. Palgrave

McLaren, B., Scheuer, O., De Laat, M., Hever, R., de Groot, R. \& Rosé, C. P. (2007). Using Machine Learning Techniques to Analyze and Support Mediation of Student EDiscussions, Proceedings of Artificial Intelligence in Education, pp 331-338, IOS Press.

Morrow, R. A \& Brown, D. D. (1994). Critical Theory and Methodology, Sage Publishers

Nijstad, B. A., \& Stroebe, W. (2006). How the group affects the mind: a cognitive model of idea generation in groups. Personality and Social Psychology Review 10(3), 186-213.

Rosé C. P., \& VanLehn, K. (2005). An Evaluation of a Hybrid Language Understanding Approach for Robust Selection of Tutoring Goals, International Journal of AI in Education 15(4), pp 325-355.

Rosé, C. P., Wang, Y.C., Cui, Y., Arguello, J., Stegmann, K., Weinberger, A., Fischer, F., (2008). Analyzing Collaborative Learning Processes Automatically: Exploiting the Advances of Computational Linguistics in Computer-Supported Collaborative Learning, submitted to the International Journal of Computer Supported Collaborative Learning 3(3), pp237-271. 
Rosé, C. P., Kumar, R., Aleven, V., Robinson, A., Wu, C. (2006). CycleTalk: Data Driven Design of Support for Simulation Based Learning, International Journal of Artificial Intelligence in Education Special Issue on The Best of ITS '04, 16, pp 195-223.

Sionti, M., Ai, H., Rosé, C. P., Resnick, L. (in press). A Framework for Analyzing Develpoment of Argumentation through Classroom Discussions, in Niels Pinkwart \& Bruce McClaren (Eds.) Educational Technologies for Teaching Argumentation Skills, Bentham Science.

Taboada, M. and Grieve, J. (2004). Analyzing Appraisal Automatically. In Proceedings of AAAI Spring Symposium on Exploring Attitude and Affect in Text, AAAI Press.

Veel, R. (2000). Language, knowledge and authority in school mathematics, in Christie, F. (Ed.) Pedagogy and the Shaping of Consciousness: Linguistic and Social Processes. Continuum

Wang, H. C., Rosé, C.P., Cui, Y., Chang, C. Y, Huang, C. C., Li, T. Y. (2007). Thinking Hard Together: The Long and Short of Collaborative Idea Generation for Scientific Inquiry, Proceedings of Computer Supported Collaborative Learning, pp 754-763, Springer.

Wang, H. \& Rosé, C. P. (2007a). A Process Analysis of Idea Generation and Failure. Proceeding of the Annual Meeting of the Cognitive Science Society, pp 1629-1634.

Wang, H. C. \& Rosé, C. P. (2007b). Supporting Collaborative Idea Generation: A Closer Look Using Statistical Process Analysis Techniques, Proceedings of Artificial Intelligence in Education, pp 659-661, IOS Press.

Weinberger A., \& Fischer F. (2006). A framework to analyze argumentative knowledge construction in computer supported collaborative learning. Computers \& Education, 46, pp 71-95. 
Whitelaw, C. and Argamon, S. (2004). Systemic Functional Features in Stylistic Text Classification. In Proceedings of AAAI Fall Symposium on Style and Meaning in Language, AAAI Press. 